\title{
LQG control of horizontal wind turbines for blades and tower loads alleviation
}

\author{
A. Pintea*, N. Christov**, D. Popescu*, P. Borne*** \\ * Automatic Control and Computer Science Faculty, University 'Politehnica' of Bucharest, 60042, \\ Bucharest, Romania (emails:andreea.pintea@gmail.com(corresponding author), \\ popescu_upb@yahoo.com) \\ ** Université Lille 1 Sciences et Technologies, 59651 Villeneuve d'Ascq, France (e-mail: \\ nicolai.christov@univ-lille1.fr) \\ **** Ecole Centrale de Lille, 59651 Villeneuve d'Ascq, France (e-mail: pierre.borne@ec-lille.fr)
}

\begin{abstract}
The paper deals with the LQG controller design optimizing the amount of power produced by two bladed horizontal variable speed wind turbines. The proposed controller ensures not only an optimal operation of turbines but also enables a compromise with the minimization of the blade oscillations and with the tower bending tendency.
\end{abstract}

Keywords: LQG control, Wind turbines, Multi-objective control

\section{INTRODUCTION}

The continuous growth of energy need together with the advantages brought by the utilization of wind turbines, made impetuous the optimization of their functioning. From a control point of view, the problem consists not only on ensuring an optimal operation, but also on load reduction and grid integration. Another important challenge is to provide good quality energy delivery from a profoundly irregular primary source, the wind. The characteristics of the wind energy source are important in different aspects regarding wind energy exploitation. The energy available in the wind varies with the cube of the wind speed. The wind is highly variable both in space and in time [Cutululis et al. 2002].

The wind speed is modelled in the literature as a nonstationary random process, yielded by superimposing two components [Cutululis et al. 2002]:

$v(t)=v_{s}(t)+v_{t}(t)$,

where $v_{s}(t)$ is the low frequency component. It describes the behaviour of the wind currents on long term, and it comprises low frequency variations of the wind. The second component, $v_{t}(t)$ is a turbulent component, that corresponds to the fast, high frequency variations. These factors make control to play an increasing role in wind turbine regulation, by a continuous essay to significantly improve all aspects of a wind energy conversion system.

Many research works deal with the control of horizontal variable speed wind turbines, starting from simple SISO techniques to multi-objective MIMO methods. In [Jelavic et al. 2009], [Cutululis et al. 2002] and also in [Camblong 2008] some solutions are proposed using PI and RST controllers. These methods, although simple and robust, do not comply with the need of building a multivariable controller and most of the times they require the implementation of multiple control loops in order to accomplish multiple control objectives.
This paper deals with the design of a LQG controller for pitch regulated variable speed wind turbines where the controller is used primarily for controlling the pitch angle through a collective pitch technique in the high wind speed in order to guarantee the power regulation. The pitch angle and electromagnetic torque control variables are used to meet specified objectives for Full Load Zone. These control objectives are to reduce structural dynamic loads and to regulate the power produced by the wind turbine. The proposed controller is designed in order to optimise a tradeoff between these objectives. By this, one tries to improve the structural integrity of the wind turbine through an estimation of the loads, and the reliability of the components, such as drive trains, blades, and tower. An advanced controller could not only ensure desired performances but will help in increasing the lifetime of the turbine and its components and in the same time in reducing the costs necessarily to perform its maintenance.

The wind turbine operation can be decomposed into several operating zones, depending on the speed of the wind that passes through the rotor. From a general point of view, in the low wind speeds regime, the turbine has to maximize the amount of power that it extracts from the wind, while in the above rated wind speed, also called high wind speeds area, the quantity of electrical power delivered has to be limited to the nominal value of the generator in order to its prevent overheating.

The paper is organised as follows: after the short introduction and presentation of the context in which an LQG regulator was chosen, one continues with Section 2 that presents in detail the mathematical model of the wind turbine. Section 3 is devoted to the design of the LQG controller and Section 4 presents an analysis of the results obtained. Section 5 includes some conclusions and perspectives. The numerical values of the turbine parameters used in the simulation are given in Annex. 


\section{WIND TURBINE SYSTEM MODELLING}

At present, there are several variable speed wind turbine configurations that are being widely used. For this study, a horizontal variable speed wind turbine was chosen. The variable speed wind turbine type is currently the most used technology and it has proven its advantages over the years [Bianchi et al. 2002]. The major advantage is that by allowing the rotor to operate at various speeds, one can obtain a more efficient capture of the wind energy with less stress in the turbine drive train during wind gusts. Different wind turbine models are presented and discussed in [Cutululis et al. 2002], [Jianlin et al. 2008], [Hau 2006]. Generally, a model for an entire wind energy conversion system can be structured as several interconnected subsystem models: an aerodynamic, a mechanical, electrical and actuator subsystems. Since the dominant dynamics lie in the mechanical subsystem, special attention will be paid to this aspect.

The mechanical structure of turbines is usually considered as being arranged into several rigid bodies linked by flexible joints. The amount of these joints or degrees of freedom, determines the order of the model. Even a few degrees of freedom can give rise to high order nonlinear models [Lupu et al. 2006]. Therefore, it is important to consider in the model just those degrees of freedom that are directly coupled to the control [Bianchi et al. 2002]. For this reason, the model presented here includes only the first mode of the drive train, the first mode of tower bending dynamics and the first mode of flapping of the blades. These degrees of freedom are sufficient for the presented controller design (Fig.1). The drive train is modelled as a two rigid bodies linked by a flexible shaft (Fig. 2). It is also supposed that the two blades move in unison and support the same forces.

Consider the general equation of motion of a mechanical system

$M \cdot \ddot{q}+C \cdot \dot{q}+K \cdot q=Q(\dot{q}, q, t, u)$

where $M, C$ and $K$ are the mass, damping and the stiffness matrices, $Q$ is the vector of the forces acting on the system and $q$ is the vector of generalised coordinates. For the turbine model, the generalised coordinates vector is

$q=\left(\omega_{T}, \omega_{G}, \zeta_{1}, \zeta_{2}, y_{T}\right)$

where $\omega_{T}$ is the angular speed of the rotor, $\omega_{G}$ stands for the angular speed of the generator, $\zeta_{1}$ and $\zeta_{2}$ are the flaps of the blades, while $y_{T}$ represents the horizontal movement of the tower (Fig.1).

As previously mentioned, we considered that $\zeta_{1}=\zeta_{2}=\zeta$ which leads to $F_{\text {aerol }}=F_{\text {aero } 2}=F_{\text {aero }}$ and $q=\left(\omega_{T}, \omega_{G}, \zeta, y_{T}\right)$. In the same time, one can find that

$Q=\left(C_{\text {aero }},-C_{e m}, F_{\text {aero }}, 2 \cdot F_{\text {aero }}\right)$

where $C_{\text {aero }}$ is the aerodynamic torque, $C_{e m}$ is the electromagnetic torque and $F_{\text {aero }}$ represents the thrust.

The aerodynamic torque and the thrust force acting on the entire rotor are expressed in terms of non-dimensional thrust and power coefficients, $C_{P}$ and $C_{T}$ respectively, as

$$
\begin{aligned}
& C_{\text {aero }}=\frac{1}{2} \cdot \rho \cdot \pi \cdot R^{2} \cdot C_{P}(\lambda, \beta) \cdot \frac{v^{3}}{\omega_{T}} \\
& F_{\text {aero }}=\frac{1}{2} \cdot \rho \cdot \pi \cdot R^{2} \cdot C_{T}(\lambda, \beta) \cdot v^{2},
\end{aligned}
$$

where $\rho$ is the air density, $R$ is the blade radius, and $v$ is the

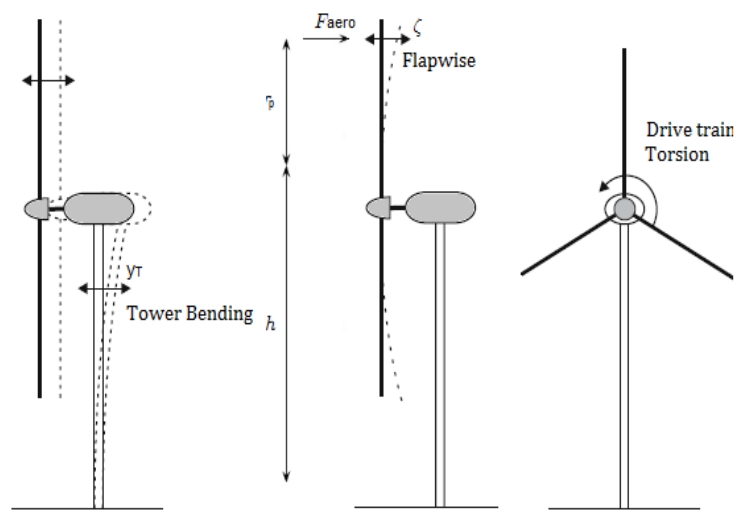

Fig. 1 Mechanical structure of the wind turbine

average speed of the wind. The power coefficient $C_{p}(\lambda, \beta)$ expresses the efficiency of the wind turbine and also characterizes the aerodynamic torque that moves the turbine rotor. The coefficients $C_{p}(\lambda, \beta)$ and $C_{T}(\lambda, \beta)$ can be represented in a polynomial form and depend on two parameters: the tip ratio of the blades $\lambda$ and the pitch angle of the blades $\beta$.

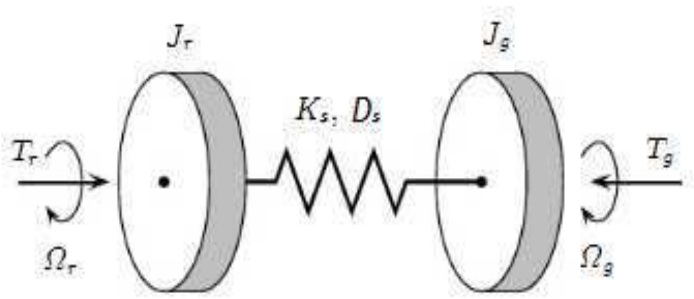

Fig. 2 Two mass model representation of the drive train

Consider the Lagrange equation

$\frac{d}{d t}\left(\frac{\delta E_{c}}{\delta \dot{q}_{i}}\right)-\frac{\delta E_{c}}{\delta q_{i}}+\frac{\delta E_{d}}{\delta \dot{q}_{i}}+\frac{\delta E_{P}}{\delta q_{i}}=Q$

where $E_{c}, E_{d}$ and $E_{p}$ denote the kinetic, the dissipated and the potential energy respectively. For the considered class of wind turbines one has

$$
\begin{aligned}
& E c=\frac{J t}{2} \cdot \omega_{T}^{2}+\frac{J_{G}}{2} \cdot \omega_{G}^{2}+\frac{M_{T}}{2} \cdot \dot{y}_{T}^{2}+M_{P} \cdot\left(\dot{y}_{T}+r_{P} \cdot \dot{\zeta}\right)^{2} \\
& E_{D}=\frac{d_{A}}{2} \cdot\left(\omega_{T}-\omega_{G}\right)^{2}+d_{P} \cdot\left(r_{P} \cdot \dot{\zeta}\right)^{2}+\frac{d_{T}}{2} \cdot \dot{y}_{T}^{2} \\
& E_{P}=\frac{k_{A}}{2} \cdot\left(\theta_{T}-\theta_{G}\right)^{2}+k_{P} \cdot\left(r_{P} \cdot \zeta\right)^{2}+\frac{k_{T}}{2} \cdot y_{T}^{2}
\end{aligned}
$$

These energies were calculated under the assumpton that the generalised force $F_{\text {aero }}$ acting on the rotor is applied on a point situated at a distance $r_{P}$ on each blade from the hub of the rotor (Fig. 1). In equations (5) $J_{t}$ and $J_{G}$ represent the rotor 
and generator moments of inertia, $M_{T}$ and $M_{P}$ are the masses of the tower and of the blade, $d_{P}$ and $d_{A}$ and $d_{T}$ represent the damping coefficients for the blade, drive shaft and the tower. Similarly, $k_{P}, k_{A}, k_{T}$ stand for the spring coefficients of the blade, drive shaft and the tower. $\Theta_{\mathrm{T}}$ and $\Theta_{\mathrm{G}}$ are the angular positions of the rotor and the generator.

The interconnection of the different plant subsystems leads to a global highly non linear system (mainly due to the nonlinearity of $\mathrm{C}_{\mathrm{aero}}$ and $\mathrm{F}_{\mathrm{aero}}$ ). For control design purpose, the global model is linearized around an operating point $\mathrm{S}_{\mathrm{op}}$ :

$$
\begin{aligned}
& C_{\text {aero }}=D_{c \omega} \cdot \omega_{T}+D_{c \beta} \cdot \beta+D_{c v} \cdot v \\
& F_{\text {aero }}=D_{f \omega} \cdot \omega_{T}+D_{f \beta} \cdot \beta+D_{f v} \cdot v
\end{aligned}
$$

where $D_{c \omega}=\left(\frac{\partial C_{\text {aero }}}{\partial \omega_{T}}\right)_{S o p}, D_{c \beta}=\left(\frac{\partial C_{a e r o}}{\partial \beta}\right)_{S o p}$,

$D_{c v}=\left(\frac{\partial C_{\text {aero }}}{\partial \nu}\right)_{S o p}, \quad D_{f \omega}=\left(\frac{\partial F_{\text {aero }}}{\partial \omega_{T}}\right)_{S o p}, \quad D_{f \beta}=\left(\frac{\partial F_{\text {aero }}}{\partial \beta}\right)_{S o p}$,

and $D_{f v}=\left(\frac{\partial F_{\text {aero }}}{\partial v}\right)_{S o p}$.

The turbine model is completed with the pitch actuator model [Lescher et al. 2005], [Nourdine et al. 2010]:

$$
\frac{\beta}{\beta_{r e f}}=\frac{1}{1+T_{\beta} \cdot s}
$$

where $\beta_{\text {ref }}$ is the desired pitch angle and $\beta$ is the actual pitch angle of the blades. Taking into account that the pitch servomotor has some physical limitations, it is modelled including saturations in the position and in the speed (Fig. 3). It is assumed that the saturation values in position are 0 and $45^{\circ}$, and that the servomotor does not exceed the speed of $10^{\circ}$ $/ s$.

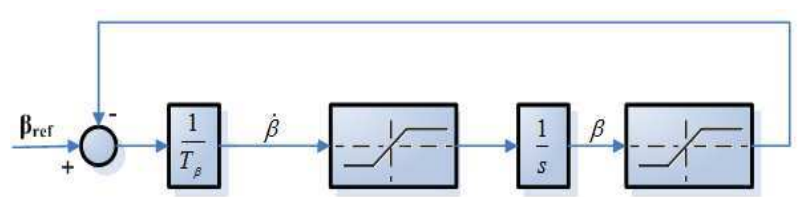

Fig.3 Pitch servomotor dynamics model

The turbulent component of the wind speed $v(t)$ passing through the rotor is also considered as a first order dynamic process disturbed by a Gaussian white noise $m_{v}(t)$ [Lescher et al. 2006], [Jianlin et al 2008]:

$$
\dot{v}(t)=-\frac{1}{T_{v}} \cdot v+m_{v}(t)
$$

with time constant $T_{v}$ calculated from the stochastic properties of the wind speed [Munteanu et al. 2007].

Combining equations (5), (6) and (7) and taking into account the measurement noise $w(t)$ (independent Gaussian white noise), one obtains the state-space representation

$$
\left\{\begin{array}{l}
\dot{x}(t)=A \cdot x(t)+B \cdot u(t)+M \cdot m_{v}(t) \\
y=C \cdot x(t)+D \cdot u(t)+w(t)
\end{array}\right.
$$

where $x(t), u(t)$ and $y(t)$ denote the system state, input and output vectors, respectively, and

$$
A=\left(\begin{array}{ccccccccc}
0 & 0 & 0 & 1 & -1 & 0 & 0 & 0 & 0 \\
0 & 0 & 0 & 0 & 0 & 1 & 0 & 0 & 0 \\
0 & 0 & 0 & 0 & 0 & 0 & 1 & 0 & 0 \\
-51.4 & 0 & 0 & -0.69 & 0.28 & 0 & 0 & -2 & 0.04 \\
268.29 & 0 & 0 & 1.46 & -1.46 & 0 & 0 & 0 & 0 \\
0 & -390.47 & 30.35 & 0.15 & 0 & -3.9 & 0.17 & -0.13 & 0.04 \\
0 & 457.14 & -242.85 & -0.21 & 0 & 4.57 & -1.42 & -0.21 & 0.07 \\
0 & 0 & 0 & 0 & 0 & 0 & 0 & -5.55 & 0 \\
0 & 0 & 0 & 0 & 0 & 0 & 0 & 0 & -0.14
\end{array}\right)
$$

$$
\begin{aligned}
B & =\left(\begin{array}{cccccccccc}
0 & 0 & 0 & 0 & 0 & 0 & 0 & 0 & 1 \\
0 & 0 & 0 & 0 & -2.44 \cdot 10^{-5} & 0 & 0 & 0 & 0 \\
0 & 0 & 0 & 0 & 0 & 0 & 0 & 5.55 & 0
\end{array}\right)^{T} \\
C & =\left(\begin{array}{ccccccccc}
0 & 0 & 0 & 1 & 0 & 0 & 0 & 0 & 0 \\
0 & 0 & 0 & 0 & 0.25 & 0 & 0 & 0 & 0 \\
0 & 0 & 1 & 0 & 0 & 0 & 0 & 0 & 0 \\
0 & 1 & 0 & 0 & 0 & 0 & 0 & 0 & 0
\end{array}\right), D=\left(\begin{array}{cccc}
0 & 0 & 10^{-5} \\
0 & 0 & 0 \\
0 & 0 & 0 \\
0 & 0 & 0
\end{array}\right)
\end{aligned}
$$$$
M=\left(\begin{array}{lllllllll}
0 & 0 & 0 & 0 & 0 & 0 & 0 & 0 & 1
\end{array}\right) .
$$

The system inputs are the average value of the wind speed, and two control variables: the pitch angle of the blades $\beta$ and the electromagnetic torque $\mathrm{C}_{\mathrm{em}}$ (Fig. 4). The wind variable

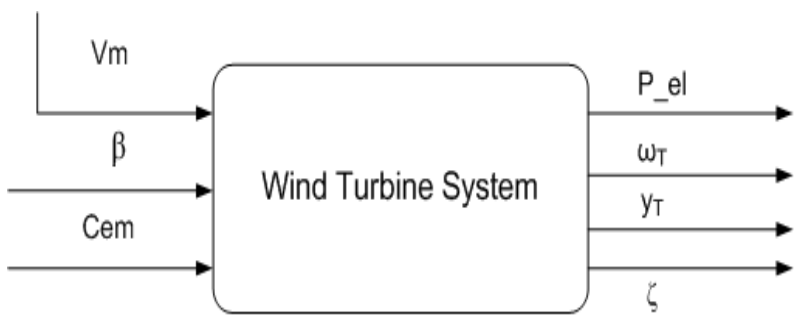

Fig. 4 Inputs and outputs of the system

included as input is seen as an external input and not as a command signal. The system state variables are $\theta_{T}-\theta_{G} \zeta, y_{T}, \omega_{T}, \omega_{G}, \dot{\zeta}, \dot{y}_{T}, \beta, v$ and the outputs are $P \_e l, \omega_{T}$, $\zeta, y_{T}$. The first output represents the electrical power generated by the turbine and can be computed as $P_{-} e l=\omega_{G} \cdot C_{e m}$. In this paper the normalized value of $P_{-} e l$ is used.

The covariance matrices of $m_{v}(t)$ and $w(t)$ are

$$
\begin{aligned}
& E\left\{m_{v}(t) \cdot m_{v}(t+\tau)^{T}\right\}=V \cdot \delta(t) \\
& E\left\{w(t) \cdot w(t+\tau)^{T}\right\}=W \cdot \delta(t) \\
& E\left\{m_{v}(t) \cdot w(t+\tau)^{T}\right\}=0
\end{aligned}
$$

with $V=\operatorname{diag}(0.1,0.5,0.1,0.1)$ and $\mathrm{W}=\operatorname{diag}(0.6,0.9,0.1$, $0.1)$.

In our case the covariance matrices were determined by taking into consideration the worst expected deviations from the mean value of a particular wind speed and the relatively low level of the measurement noises. To analyse the controller robustness, several tests with different covariance matrices have been done.

In the next section, a LQG controller is designed, having as main goal to regulate the rotor speed and to maintain the output power of the turbine to the nominal value that will not 
damage the generator, and aiming in the same time to reduce the blade flaps and the bending of the tower.

\section{DESIGN OF LQG CONTROLLER}

As it is well-known, the LQG controller is a combination of a Kalman filter and a linear-quadratic regulator defined by a gain matrix $K$ [Kwakernaak et al. 1972], [Naidu 2002]. Because the system outputs have to closely track the imposed references, a LQG controller with integral action is used for the considered wind turbine system (Fig.5). The role of the

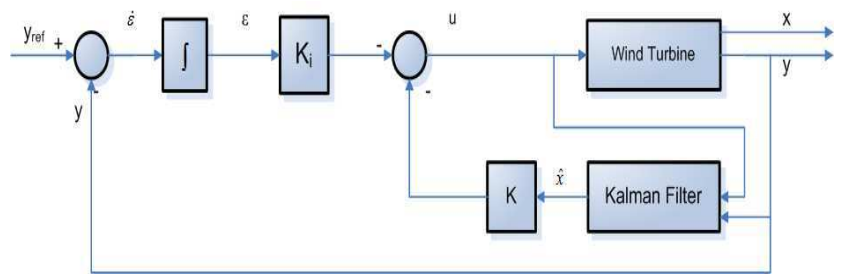

Fig. 5 General scheme of the closed-loop system with LQG controller and integral action

integral action is to cancel the tracking error defined as $\dot{\varepsilon}=y_{\text {ref }}-y$, where $\mathrm{y}_{\text {ref }}$ denotes the desired system output.

The LQG controller design is done in two major steps. First, a state feedback $u=-K_{a} z$ is determined which minimizes the cost function

$J=\int_{0}^{\infty}\left(z^{T} \cdot Q \cdot z+u^{T} \cdot R \cdot u+2 \cdot z^{T} \cdot N \cdot u\right)$

where $Q \geq 0$ and $R \geq 0$ are weighting matrices, $z=\left[\begin{array}{ll}x^{T} & \varepsilon^{T}\end{array}\right]^{T}$ and $\varepsilon$ is the integrator output (Fig.5). Denoting $K_{a}=\left[\begin{array}{ll}K & K_{i}\end{array}\right]$, the state feedback can be represented as $u=-K \cdot x-K_{i} \cdot \varepsilon$.

It is well known that $K_{a}=R^{-1} \cdot\left(B^{T} \cdot P+N^{T}\right)$, where $P \geq 0$ is the positive semi-definite solution of the Riccati equation

$A^{T} \cdot P+P \cdot A-(P \cdot B+N) \cdot R^{-1} \cdot\left(B^{T} \cdot P+N^{T}\right)+Q=0$.

In our case $Q, R$ and $N$ were chosen as $Q=\operatorname{diag}(0.1,0.2,0.8$, $0.4,0.6,0.2,0.8,0.1,0.1,0.2,0.2,0.1,0.1), R=0.4 \mathrm{I}_{2}$ and $N=\left(\begin{array}{ccccccccccccc}0.1 & 0.2 & 0.1 & 0.1 & 0.2 & 0.2 & 0.1 & 0.1 & 0.1 & 0.2 & 0.2 & 0.1 & 0.1 \\ 0.01 & 0.02 & 0.04 & 0.01 & 0.2 & 0.2 & 0.1 & 0.1 & 0.1 & 0.2 & 0.2 & 0.1 & 0.1\end{array}\right)$.

The turbine state vector $x$ being inaccessible, a Kalman filter (state estimator) is designed in the second step, in order to obtain the optimal estimate $\hat{x}$ of the state vector $x$ [Anderson et al. 1990]. The Kalman filter is described as

$\left\{\begin{array}{l}\dot{\hat{x}}=A \cdot \hat{x}+B \cdot u+K_{f} \cdot(y-C \cdot \hat{x}-D \cdot u) \\ K_{f}=P_{f} \cdot C^{T} \cdot W^{-1}\end{array}\right.$

where $P_{f}$ is the positive semi-definite solution of the Riccati equation

$P_{f} \cdot A^{T}+A \cdot P_{f}-P_{f} \cdot C^{T} \cdot W^{-1} \cdot C \cdot P_{f}+M \cdot V \cdot M^{T}=0$.

The optimal control is thus realized as

$u=-K \cdot \hat{x}-K_{i} \cdot \varepsilon$.

\section{SIMULATION RESULTS}

The control system simulations were done using MATLAB/SIMULINK software. The chosen operating point for the linearization of the system corresponds to the average value of the wind speed of $17 \mathrm{~m} / \mathrm{s}$. The numerical values of the parameters used in simulation are given in Annex.

The turbulent component of the wind speed considered as system perturbation, is generated by feeding a suitable shaping filter $H_{F}$ with a white noise [Holmes et al.2003], [Munteanu et al. 2007]. In this paper we use the filter proposed in [Nichita et al. 2000]:

$$
H_{F}(s)=K_{F} \cdot \frac{m_{1} \cdot T_{F} \cdot s+1}{\left(T_{F} \cdot s+1\right) \cdot\left(m_{2} \cdot T_{F} \cdot s+1\right)}
$$

with $T_{F}=11.36, K_{F}=4.11, m_{1}=0.4$ and $m_{2}=0.25$ calculated according to [Munteanu et al. 2007].

The wind profile used for these simulations is shown in Fig. 6. Fig. 7 and Fig. 8 show that the LQG controller succeeds to minimize the error between the perturbed and nominal values of system outputs. In turn, Fig. 9, and Fig. 10 illustrate the performances in terms of mechanical solicitations of the wind turbine tower and blades. The regulator maintains the values

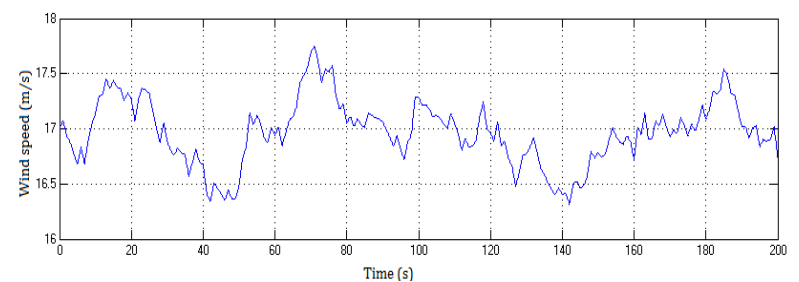

Fig.6 Wind speed profile

of the output power and of the rotational speed of the rotor respectively, around the imposed nominal values. The LQG controller was tested for various wind speeds, and the differences were insignificant. In Fig. 11 the output power curve is given obtained for wind speeds varying from 12 to 25 $\mathrm{m} / \mathrm{s}$. This demonstrates the robustness of the controller and shows that it can be used in the entire functioning zone. Note that good results were also obtained for wind speeds lower than $12 \mathrm{~m} / \mathrm{s}$. However, these results are not presented here since in this case the control objectives are different and the pitch technique is not used in industrial installations.

\section{CONCLUSIONS}

In this paper, a LQG controller design was presented for horizontal variable wind speed turbines. The Full Load zone was considered for which the control objective is to ensure a compromise between the desired performances and the need to reduce the mechanical solicitations perceived by the structure. This can be seen as a compromise between maintaining a constant rotational speed of the rotor and also of the produced electrical power to the nominal value, from one side, and from the other side, the reduction of the bending of the tower and also of the blade flapping. The obtained results demonstrate that this type of controller allows the alleviation of the mechanical stress and it ensures good performances for the entire above rated wind speeds regime. 


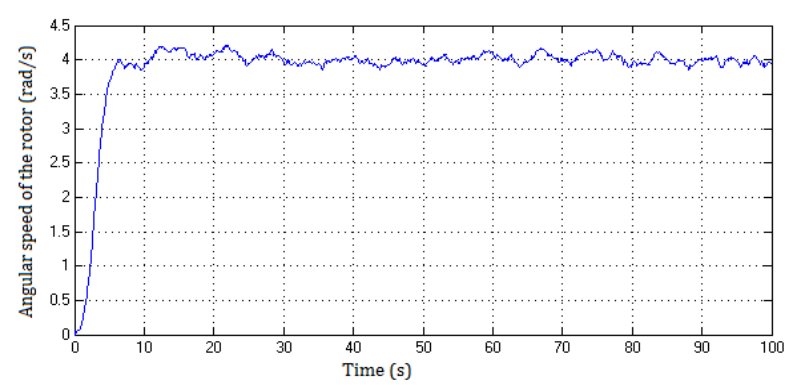

Fig. 7 Angular speed of the system with LQG controller

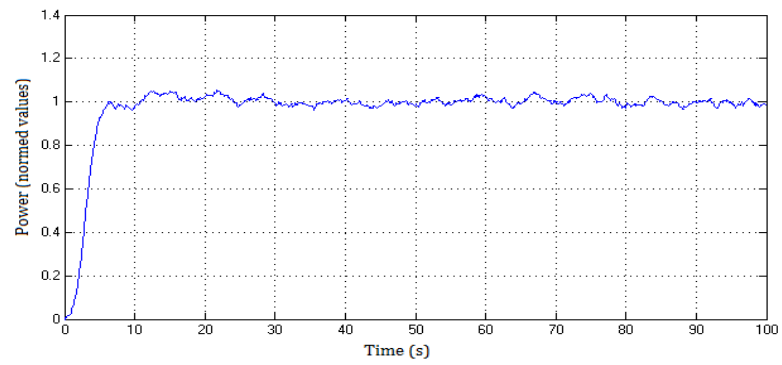

Fig. 8 Electrical power of the system with LQG controller

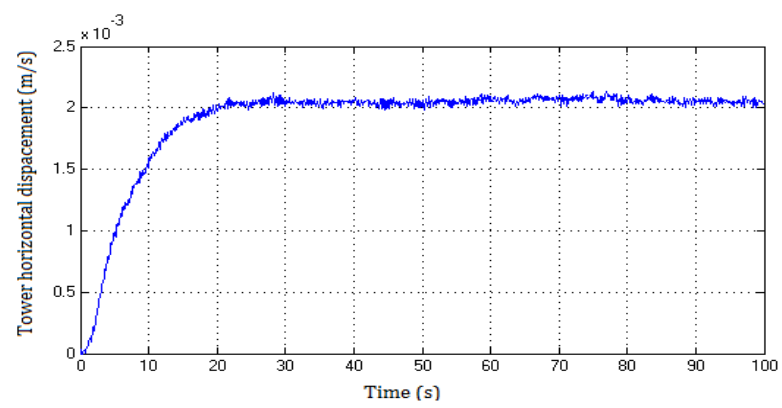

Fig. 9 Tower top horizontal displacement

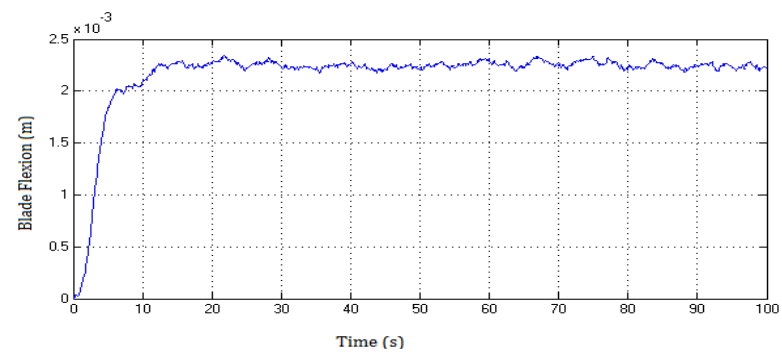

Fig. 10 Blades flexion movement

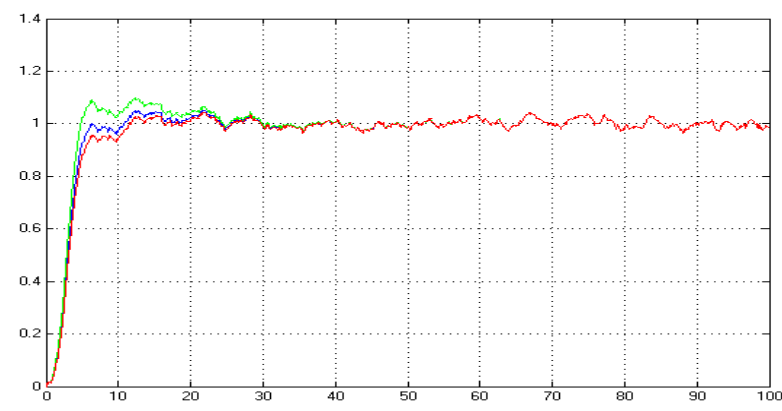

Fig. 11 Output power responses for wind speeds equal to $12 \mathrm{~m} / \mathrm{s}$ (red), $18 \mathrm{~m} / \mathrm{s}$ (blue) and $25 \mathrm{~m} / \mathrm{s}$ (green)

\section{ACKNOWLEDGMENT}

This work was partially supported by CATIIS POSDRU Project No. 32379/2009 and POSDRU7713 project.

\section{REFERENCES}

Anderson, B., D., O., Moore, J., B., "Optimal control: Linear quadratic methods", Prentice Hall, Englewood Cliffs, NJ, 1990.

Bianchi, D. F., Battista, H., "Wind turbine control systemsprinciples, Modelling and gain scheduling design", Springer-Verlag, London, 2002.

Camblong, H., "Digital robust control of a variable speed pitch regulated wind turbine for above rated wind speeds", Control Engineering Practice, Volume 16, Issue: 8, pp. 946-958, 2008.

Cutululis, N.A., Munteanu, I., Ceanga, E, Culea, M., "Optimal control structure for variable speed wind power system", The annals of "Dunarea de Jos" University of Galati, Fascicle III, pages 95-102, 2002.

Dauphin-Tanguy, G., Foulloy, L., Popescu, D., "Modélisation, identification et commande des systèmes", Editura Academiei Romane, pages 313 -348, Bucuresti 2004.

Hau, E., "Wind turbines, fundamentals, technologies, application economics", $2^{\text {nd }}$ edition, Springer-Verlag, pp. 103, 2006.

Holmes, J.D., Wind loading of structures, Spon Press, 2003.

Jelavic, M., Peric, N., "Wind turbine control for highly turbulent winds", Automatica, Vol. 50, No. 3-4, pages 135-151, 2009.

Jianlin L, Hongyan, X, Lei, Z, "Disturbance accommodating LQR method based pitch control strategy for wind turbines", Proceedings of the Second International Symposium on Intelligent Information Technology Application, Shanghai, 21-22 December, 2008, pp. 766771.

Kwakernaak, H. Sivan, R., "Linear optimal control systems", Wiley-Interscience, New York, 1972.

Lescher, F., Borne, P., "Robust gain scheduling controller for pitch regulated variable speed wind turbine", Studies in Informatics and Control, vol. 14, No. 4, pages 299-315, 2005.

Lescher, F., Zhao, J-Y, Borne, P., "Switching LPV controllers for a variable speed pitch regulated wind turbine", International Journal of Computers, Communications and Control, No.4, pages 75-86, Vol.1, 2006.

Laroche, E. "Commande Optimale", Thèse Master Images, Robotique et ingénierie pour le vivant parcours automatique et robotique, Strasbourg, 2009-2010.

Lupu, L, Boukhezzar, B., Siguerdidjane, "Pitch and torque control strategy for variable speed wind turbines", Proceedings EWEC, 2006, Athens

Munteanu, I., Ceanga, E., Optimal control of wind energy systems -Towards a global approach, Springer, 2007.

Naidu, S.D., "Optimal control systems", CRC Press, 2002.

Nichita, C., Luca, D., Dakyo, B., Ceanga, E., Cutululis, N.A., "Modelling non-stationary wind speed for renewable energy systems control", The annals of "Dunarea de Jos” University of Galati, Fascicle 3, pages 29-34, 2000. 
Nourdine, S., Camblong, H., Vechiu, I., Tapia, G., "Comparison of wind turbine LQG controllers using individual pitch control to alleviate fatigue loads", Proceedings of the Mediterranean Conference on Control and Automation, Marrakech, Morocco, June 23-25, 2010.

\section{ANNEX: PARAMETER VALUES USED IN DESIGN}

\section{AND SIMULATION}

$\mathrm{R}=17 \mathrm{~m} ; \mathrm{r}_{\mathrm{p}}=8 \mathrm{~m} ; \mathrm{Pn}=400 \mathrm{~kW} ; \omega_{\mathrm{n}}=4 \mathrm{rad} / \mathrm{s} ; \mathrm{J}_{\mathrm{T}}=214 \mathrm{Tm}^{2} ; \mathrm{J}_{\mathrm{G}}=$ $41 \mathrm{Tm}^{2} ; \mathrm{M}_{\mathrm{T}}=35 \mathrm{~T} ; \mathrm{M}_{\mathrm{P}}=3 \mathrm{~T} ; \mathrm{d}_{\mathrm{A}}=60 \mathrm{Tm}^{2} / \mathrm{s} ; \mathrm{d}_{\mathrm{P}}=10 \mathrm{Tm}^{2} / \mathrm{s}$; $\mathrm{d}_{\mathrm{T}}=50 \mathrm{Tm} / \mathrm{s} ; \mathrm{k}_{\mathrm{A}}=11000 \mathrm{Tm}^{2} / \mathrm{s}^{2} ; \mathrm{k}_{\mathrm{P}}=1000 \mathrm{Tm}^{2} / \mathrm{s}^{2} ; \mathrm{k}_{\mathrm{T}}=$ $8500 \mathrm{Tm}^{2} / \mathrm{s}^{2}$ 